\title{
Dominique Goncalvès
}

\section{Los doce primeros años de la Junta Económica y de Gobierno del Real Consulado de La Habana}

En La Habana, el viernes 10 de abril de 1795, se sentaron catorce criollos alrededor de una mesa presidida por el capitán general de la isla de Cuba. Con este acto se dio inicio a la primera sesión de la Junta Económica y de Gobierno del Real Consulado; el cual se hallaba compuesto tanto por este grupo como por un Tribunal de Comercio, destinado a reglamentar las relaciones comerciales. Las siguientes páginas se ocuparán de la Junta, sin restar importancia al Tribunal, debido a que los pocos miembros de éste eran provenientes de aquélla, y sobre todo porque el rey le confió objetivos de mayor alcance, tales como el fomento de la agricultura y del comercio, así como de la isla en general; siendo precisamente estos objetivos los que llevaron a denominarla más comúnmente como Junta de Fomento. La fundación del Consulado de La Habana se incorporó a un vasto plan de reformas comerciales del imperio español, impulsado por la Corona a partir de 1778. Le precedieron por algunos meses solamente el Consulado de Caracas y el de Buenos Aires, y muy poco después se erigieron los de Veracruz, Guadalajara, Cartagena y Montevideo.

La originalidad del Consulado de La Habana consistía en que, desde el inicio, el fomento esperado se debía al cultivo a gran escala de la caña de azúcar que rendía mucho dinero. En cierto modo, fue esta planta la que presidió la creación de aquella institución. Su producción en Cuba es antigua, pero no cobró importancia sino hasta las reformas económicas desarrolladas a partir de 1763-1765, cuando al final de la guerra de los Siete Años Inglaterra devolvió el dominio de La Habana a España. La independencia de los Estados Unidos en 1776 transformó el juego económico en el Caribe, lo que permitió incrementar aún más la producción de caña. Sin embargo, el inicio de la década de 1790 marcó una etapa decisiva por la repentina aparición de un mercado mundial del azúcar a conquistar, en la que España se encontraba en la mejor posición. A ello contribuyó el hecho de que las 
plantaciones de la isla de Haití, que era hasta 1791 el primer productor mundial de azúcar, habían desaparecido en algunas semanas por los incendios provocados por los esclavos sublevados. En estas circunstancias, todo el mundo sabía que el precio del azúcar iba a aumentar mucho.

Otro elemento determinante para la creación de la Junta fue la existencia en La Habana de una oligarquía de hacendados, constituida desde hacía tiempo, capaz de desempeñar la función directiva de este organismo. Esta oligarquía ya era poderosa en La Habana: políticamente, por su control del Cabildo, y financieramente, por los beneficios extraordinarios generados por el azúcar. A esta élite, que ya se llamaba sacarocracia, hay que sumar un grupo de comerciantes enriquecidos por la importación de esclavos y de todos los productos necesarios en una isla donde las producciones eran cada vez más especializadas, así como por la exportación de azúcar o café. Hay que entender a estas élites en un sentido familiar más que individual. Algunas familias descendían de los primeros conquistadores de la isla, tenían algunas veces títulos de Castilla, y todas estaban integradas en redes de solidaridad americanas y transatlánticas. El rey eligió dentro de esta oligarquía a las familias que quería que integraran la primera composición de la Junta. Son ellas las que propusieron la creación de esta institución, con un objetivo sencillo: hacer dinero para ellos y para la Monarquía. Era un deseo compatible con los propósitos de la Corona. El gran crecimiento económico y el fomento de la colonia debían significar ingresos importantes a la metrópoli, además de atar más estrechamente a los dos territorios. De momento, con el Consulado se iniciaron nuevas relaciones entre los poderosos habaneros y la Corona española.

Las líneas que siguen tratan de aportar algunas respuestas a la siguiente pregunta: ¿Qué papel desempeñó la Junta Económica y de Gobierno del Real Consulado en la relación entre la oligarquía y la administración real entre 1795 y 1807 ? Para entender las negociaciones entre las élites y la Corona nos centraremos en dos puntos de reflexión. El primero trata del poder del Consulado, el que le confirió el rey y el que asimismo podía provenir de la oligarquía que lo componía. En el segundo analizaremos las negociaciones entre la Junta Económica y de Gobierno y el rey o sus representantes. Esto nos permitirá 
ver cuáles eran los asuntos pendientes, con qué fuerzas contaba cada uno, y cuáles fueron los logros para las dos partes.

La razón que incita a privilegiar el estudio de estos doce primeros años viene esencialmente de un problema de fuentes. La reflexión elaborada aquí proviene en gran parte del examen de algunos legajos de los archivos del Real Consulado de La Habana y particularmente de las actas de las sesiones de dicha institución. ${ }^{1}$ Se cubren así todos los años de vida del Consulado menos el periodo que va de junio de 1807 hasta abril de 1812, por haber desaparecido los libros. Por otro lado, los primeros años, marcados por el entusiasmo de los inicios, siempre se han visto como parte de un periodo de cambios decisivos en la historia de Cuba, por lo que han merecido un estudio particular que nos indujo a detenernos en 1807. Algunos documentos conservados en otros depósitos cubanos, como el Archivo de la Oficina del Historiador de la ciudad de La Habana, el Instituto de Historia y Lingüística, y también en el Archivo General de Indias de Sevilla, tratan de complementar a estas fuentes principales.

\section{La aparición de una pieza maestra en el ajedrez político}

Francisco de Arango y Parreño desempeñó hasta su muerte en 1837 un papel determinante en todas las relaciones entre la colonia y la metrópoli. Negoció en Madrid, como apoderado de la ciudad de La Habana, los artículos de la Junta con el secretario de Estado, Diego María de Gardoqui y Arrequibar, ${ }^{2}$ quien redactó la cédula de formación. La figura de Francisco de Arango y Parreño es bien conocida; proveniente de una de las más antiguas y poderosas familias de La Habana -por ejemplo, su abuelo fue director de la Compañía de Tabacos- fue abogado y muy tempranamente habría de poner sus eminentes talentos retóricos al servicio de la oligarquía de hacendados habaneros. Su Discurso sobre la agricultura de la Habana y medios de

1 Los ejemplos mostrados aquí provienen de los Libros de actas de sesiones de la Junta de Gobierno, conservados en el Archivo Nacional de Cuba en La Habana. Para saber a qué libro precisamente se refieren, ponemos aquí las referencias exactas, y las fechas que cubre cada libro: libro 161: abril 1795 a julio 1796; libro 162: agosto 1796 a noviembre 1797; libro 163: diciembre 1797 a julio 1799; libro 164: agosto 1799 a diciembre 1801; libro 165: enero 1802 a junio 1803 ; libro 166: julio 1803 a junio 1805; libro 167: julio 1805 a mayo 1807.

2 ANC, Real Consulado y Junta de Fomento, leg. 1, exp. 1. 
fomentarla, publicado en $1792,{ }^{3}$ fue el vibrante alegato que indujo a la Monarquía a fundar el consulado.

Hubo anteriormente dos intentos de promover un consulado en $\mathrm{La}$ Habana. El primero, en 1783, fue promovido por el cuerpo de comerciantes de La Habana (Arregui 1983). El segundo tuvo lugar en 1787, cuando el Cabildo quiso hacer un consulado "a imitación del de Santander" (Tornero Tinajero 1996). Quizás los dos intentos fracasaron porque provinieron de categorías sociales entonces opuestas, que no reunían la fuerza necesaria para lograrlo.

\section{Mucho poder, pero controlado}

El reglamento interno ${ }^{4}$ del Consulado se inspiró de cierta forma en el del Consulado de Bilbao. La composición era clásica: un prior, dos cónsules, nueve consejeros y un síndico, haciendo un total de trece cargos electivos; sumaban de hecho veintiséis personas ya que cada cargo tenía un propietario y su teniente. Todas estas personas tenían derecho de proponer proyectos y debían votar a favor o en contra de las proposiciones. Las plazas se repartían por igual entre hacendados y comerciantes. A este grupo hay que añadir un tesorero, un secretario, un contador y un escribano, y también el personal auxiliar.

Como en cada asociación ya existente en la isla, la autoridad suprema estaba confiada al capitán general, quien era su presidente nato, y salvo el intendente o sus tenientes respectivos, ningún otro alto funcionario tenía el derecho de presidir la Junta. Las demás asociaciones o instituciones debían prestarle asistencia en caso de que lo pidiera. En las ciudades de Puerto Príncipe, Trinidad, Santo Espíritu, Villa Clara, San Juan de los Remedios, Matanzas, Santiago de las Vegas, Santiago de Cuba, Bayamo y Baracoa, el Consulado estaba presente a través de diputaciones. También la jurisdicción del Consulado abarcaba toda la isla. La Junta contaba con importantes medios para realizar su tarea. El primero de los cuales era la delegación por el rey del derecho de avería. Este impuesto correspondía al $0,5 \%$ del valor de cada mercancía importada o exportada por cada puerto de la isla. La segunda fuente de ingresos estaba constituida por todas las multas fijadas por el nuevo Tribunal de Comercio, más las del ya existente Tribunal de

Fue reeditado en Arango y Parreño (1952, I: 114-162).

ANC, Real Consulado y Junta de Fomento, leg. 1, núm. 1. 
Alzadas. Se integraba esta Junta mediante una elección, que de facto era una cooptación. Para postular, el reglamento imponía a los pretendientes gozar de una buena reputación, poseer una buena fortuna, tener competencias probadas en materia de agricultura o de comercio; dejando en manos de los que ya eran miembros la decisión de quienes llenaban estos requisitos.

Por otra parte, la Monarquía prefería tomar medidas para que no se votaran decisiones en contra de sus intereses, más que tener que suspenderlas después. Existía un eficaz control del organismo, fundado tanto en una limitación escrita de los poderes como en un control de los miembros. El poder estaba limitado en el tiempo, dado que los trece cargos de decisión eran bienales. Como en otras instituciones existentes, las elecciones debían ser avaladas por el rey. No estaba permitido que hubiera candidatos, $\mathrm{y}$ un doble sorteo determinaba tanto a los electores como a los elegidos. Era lógico que en tal sistema hubiera gente elegida en contra de su voluntad. Estaba prohibido rechazar la elección. Tampoco se podía reunir la Junta si no había un mínimo de seis personas. Por fin, una excepción fue hecha para el fiel Francisco de Arango: el rey le confió el puesto clave de síndico por un tiempo que dependía de su real voluntad, asignándole 3.300 pesos mensuales y nombrándolo oidor honorario de la Audiencia de Santo Domingo. Hay que notar que a diferencia por ejemplo del Consulado de Caracas, donde el prior y los cónsules recibían algunos emolumentos (Arregui 1981), en el de La Habana, ninguno de los vocales percibió salario. Este arsenal de disposiciones estaba destinado a impedir que un grupo demasiado pequeño se apoderara de la institución.

Aunque no se ganaba sueldo siendo vocal del Consulado de $\mathrm{La}$ Habana, para suscitar fidelidades y un trabajo bien hecho, la Monarquía atraía con reales recompensas, tal y como lo dice el párrafo 21 :

$\mathrm{Y}$ los que den mayores pruebas de amor a mi servicio, y a la felicidad de mis vasallos [...], y dexen señalado su tiempo con algún beneficio particular de la agricultura, y del comercio, me hallarán siempre dispuesto a premiarlos, y condecorarlos, según su mérito y circunstancias. Para lo cual encargo al capitán general esté siempre atento a lo que hagan y trabajen por la causa pública cada uno en su tiempo: y que cumplido éste me informe de ello de oficio, y con reserva, oyendo antes la calificación del síndico.

Es sabido que el Antiguo Régimen daba espacio a distinciones honoríficas y también que el servicio del Estado obedecía a reglas de 
reclutamiento y de promoción muy particulares. Entonces, lograr primero ser miembro de la Junta, y segundo distinguirse por la eficacia del trabajo, permitía el acceso a honores o podía favorecer carreras ajenas al consulado; lo cual, con el tiempo, podía traer mucho dinero. La novedad del Consulado de La Habana residía en el hecho de que ahora los comerciantes tendrían una institución que podría premiarlos, dado que el Cabildo estaba casi totalmente controlado por los hacendados.

\section{Una pieza maniobrada por una oligarquía}

La historiografía ha mostrado en qué medida el estudio de las redes permite entender el funcionamiento de las instituciones (Castellano/ Dedieu 1998). El Consulado de La Habana no escapa a la regla.

Presentamos a continuación la lista de la primera composición de la Junta de Fomento, tal como fue confeccionada en Madrid después de algunas reflexiones, entre las cuales las sugerencias de Francisco de Arango y Parreño fueron determinantes (Arregui 1983).

Cuadro 1: Composición de la primera

Junta de Gobierno del Real Consulado, 1795-1797

\begin{tabular}{lll}
\hline Cargo & Titular & Teniente \\
\hline Prior (h) & Conde de Casa Montalvo & Marqués del Real Socorro \\
Cónsul 1 (c) & Juan Tomás de Jáuregui & Manuel José Torrontegui \\
Cónsul 2 (c) & Lorenzo de Quintana & Juan Francisco de Oliden \\
$1^{\circ}$ Consejero (h) & Marqués Real Socorro & José Cipriano de la Luz \\
$2^{\circ}$ Consejero (h) & Marqués de Villalta & José Ricardo O’Farrill y \\
& & Herrera \\
$3^{\circ}$ Consejero (c) & Juan Bautista Lanz & Pedro Martín de Alguer \\
$4^{\circ}$ Consejero (c) & Pedro Juan de Erice & Mariano Carbo \\
$5^{\circ}$ Consejero (h) & Marqués de Casa Peñalver & Pedro Regalado de Pedroso \\
$6^{\circ}$ Consejero (h) & Marqués de Arcos & Miguel de Cárdenas y Santa \\
& & Cruz \\
$7^{\circ}$ Consejero (c) & Manuel de Quintanilla & Pablo Boloix \\
$8^{\circ}$ Consejero (c) & Antonio de Arregui & Bernabé Martínez de Pinillos
\end{tabular}

Esta persona nunca tomó posesión de su cargo, por haber fallecido antes de la primera sesión. Fue reemplazada por Pablo Boloix, su teniente. 


\begin{tabular}{lll}
\hline Cargo & Titular & Teniente \\
\hline $9^{\circ}$ Consejero (h) & $\begin{array}{l}\text { Nicolás Calvo de la } \\
\text { Puerta y O'Farrill }\end{array}$ & Agustín de Ibarra \\
Síndico (h) o (c) & $\begin{array}{c}\text { Francisco de Arango y } \\
\text { Parreño }\end{array}$ & Antonio de Robredo \\
& Antonio del Valle Hernández \\
Secretario & Ramón de Arango y Parreño \\
Contador & Rafael de Armas & \\
Tesorero & Santiago de Arandia & \\
Escribano &
\end{tabular}

(h) indica que el cargo debía ser ocupado por un hacendado y (c), por un comerciante.

Se pueden dar algunos detalles sobre las personas aquí mencionadas y sus familias, y más sobre las familias de hacendados que sobre las de comerciantes; ciertamente los terratenientes accedieron a una nobleza -o pudieron probar su pertenencia a la misma-, la que consiguientemente incitó a la producción de trabajos genealógicos. Las personas o las familias que la Corona quiso distinguir fueron electas de entre las que tenían un fuerte capital de tierras o financiero, duplicándose por un fuerte capital de relaciones. Esta era una de las condiciones necesarias para el funcionamiento de la institución. Estas familias habían probado que dominaban perfectamente los asuntos de plantación o de comercio mediante la posesión de grandes ingenios o grandes almacenes; y su influencia debía dar más fuerza a las decisiones tomadas. No es posible hablar aquí de todo el haber patrimonial de las familias, ni reconstituir las redes de sus negocios, pero es imprescindible dar algunos datos biográficos.

Empezamos por los hacendados: el prior, el conde de Casa Montalvo, era hijo del primer conde de Macuriges, antiguo intendente de Marina de La Habana, que se distinguió por su acción heroica en la toma de La Habana por los ingleses en 1762. El conde de Casa Montalvo era en 1794 brigadier, así como caballero de la Orden de Santiago, y tenía los honores de gentilhombre de Cámara. El teniente de prior (y también consejero) ostentaba el título de marqués del Real Socorro por los importantes servicios dados a la Monarquía. El título de marqués de Villalta fue dado en 1668, y era uno de los más antiguos ostentado en Cuba; ya se puede imaginar todo el honor que recaía sobre su propietario, José Luis de Herrera y Chacón. El marqués de Casa Peñalver provenía de una de las familias más poderosas y ambi- 
ciosas, dado que su padre había aceptado secundar en la administración de la isla al almirante inglés que ocupó militarmente La Habana. La reconciliación del marqués con la Monarquía fue oficializada por la concesión de este título en 1790. Pedro Regalado de Pedroso y Zayas, pertenecía a la que seguramente era la más antigua familia de $\mathrm{La}$ Habana, llegada a finales del siglo XVI. El segundo marqués de Arcos se llamaba José de Peñalver y Navarrete, y por lo tanto formaba parte del poderoso clan de los Peñalver. Su teniente, el coronel Miguel de Cárdenas y Santa Cruz, era el hijo del primer marqués de Cárdenas de Montehermoso; también éste debía su título a su actitud frente al invasor inglés. Nicolás Calvo de la Puerta y O'Farrill era el hijo del primer conde de Buenavista.

Ya hemos visto por qué Francisco de Arango y Parreño fue electo. La mención de estos apellidos, que encontramos en un orden diferente en muchos personajes, supone parentescos. Sobre este tema igualmente la investigación genealógica da informaciones preciosas. En el caso de los hacendados, el grado de parentesco era cercano. Generalmente eran primos, suegro y yerno, a veces hermanos, como se observa entre los Arango y Parreño. También el marqués de Arcos era primo hermano del marqués de Casa Peñalver, quien era el suegro de Miguel de Cárdenas Santa y Cruz. El marqués de Villalta era primo hermano de José Ricardo O'Farrill y Herrera, primo también del conde de Casa Montalvo, y primo político de Pedro Regalado de Pedroso.

Detengámonos ahora en los comerciantes. El primer cónsul, Juan Tomás de Jáuregui, era el cuñado de Agustín Jáuregui Aldecoa, virrey del Perú. El segundo cónsul, Lorenzo de Quintana, era apoderado del Ayuntamiento de Cádiz en La Habana ${ }^{6}$ y comerciaba con La Florida. Pedro Juan de Erice era uno de los más grandes comerciantes de La Habana. El caso del comerciante Bernabé Martínez de Pinillos es bien conocido: llegado a Cuba en la década de 1770 había fundado una casa de comercio, empezando por traficar de todo, ${ }^{7}$ para convertirse con el tiempo en uno de los más grandes negreros de la plaza. Es lamentable que la documentación no permita conocer los lazos de parentesco de los comerciantes. Sin embargo existían entre ellos lazos

6 ANC, Protocolos Notariales, Escribanía de Salinas, leg. 861, exp. 10.395; leg. 863, exp. 10.433; y leg. 866, exp. 10.495. Esta referencia y la siguiente fueron facilitadas al autor por Manuel Barcia Paz.

7 ANC, Protocolos Notariales, Protocolo de Hacienda 1786-1790, f. 59r y f. 353r. 
de naturaleza comercial. En efecto, la mayoría de las personas citadas aquí formaban parte de la Compañía gaditana de negros.

En suma, el Consulado era básicamente un asunto de familia y lo seguiría siendo durante mucho tiempo, dado que el modelo de elección estaba fundado en la cooptación. Miremos por ejemplo la composición del Consulado en junio de 1803, o sea siete elecciones después de la composición de la primera Junta

Cuadro 2: Composición de la Junta de Fomento del Real Consulado del $1^{\circ}$ de junio de 1803

\begin{tabular}{|c|c|c|}
\hline Cargo & Titular & Teniente \\
\hline Prior (h) & Marqués de Casa Peñalver & Conde de Zaldívar \\
\hline Cónsul 1 (c) & Francisco de Santa Cruz & $\begin{array}{l}\text { Bonifacio González Larri- } \\
\text { naga }\end{array}$ \\
\hline Cónsul 2 (c) & José Antonio de Arregui & Juan José de Yguarran \\
\hline $1^{\circ}$ Consejero $(\mathrm{h})$ & $\begin{array}{l}\text { Marqués de Cárdenas de } \\
\text { Montehermoso }\end{array}$ & Pedro Regalado de Pedroso \\
\hline $2^{\circ}$ Consejero $(\mathrm{h})$ & Domingo de Herrera & Rafael Montalvo \\
\hline $3^{\circ}$ Consejero (c) & Juan de Santa María & Joaquín Pérez de Urria \\
\hline $4^{\circ}$ Consejero (c) & Tomás de la Cruz Muñoz & Juan de Cavo \\
\hline $5^{\circ}$ Consejero (h) & Francisco de Montalvo & Rafael O'Farrill \\
\hline $6^{\circ}$ Consejero (h) & José María Escobar & Dr. Antonio Morejón y Gato \\
\hline $7^{\circ}$ Consejero (c) & Enrique Amorrosta & Pablo Serra \\
\hline $8^{\circ}$ Consejero (c) & Felipe Fernández de Silva & Pedro de la Cuesta Manzanal \\
\hline $9^{\circ}$ Consejero (h) & Miguel de Herrera Pedroso & José Echegoyen \\
\hline Síndico (h) o (c) & $\begin{array}{l}\text { Francisco de Arango y } \\
\text { Parreño }\end{array}$ & Andrés de Jáuregui \\
\hline Secretario & $\begin{array}{l}\text { Antonio del Valle Hernán- } \\
\text { dez }\end{array}$ & \\
\hline Contador & Ciriaco de Arango & \\
\hline Tesorero & José Rafael de Armas & \\
\hline
\end{tabular}

(h) indica que el cargo debía ser ocupado por un hacendado y (c), por un comerciante.

Puestas las razones ya mencionadas, nos ocuparemos aquí únicamente de los hacendados. Nos encontramos inmediatamente con apellidos conocidos: Peñalver, Cárdenas, Pedroso, Herrera, Montalvo, O'Farrill. El segundo marqués de Cárdenas de Montehermoso, sucedió a su hermano Gabriel María Cárdenas de Santa Cruz, fallecido en 
1801. Parece solamente haber un nombre nuevo, el del conde de Zaldívar de la Real Fidelidad: el cuñado del prior, marqués de Peñalver.

Sin embargo, más tarde nos encontramos con nuevos apellidos. En junio de 1805 es elegido conciliario José Fuertes, sobre quien las fuentes no dicen nada, y se puede afirmar que no tiene ningún lazo matrimonial con la oligarquía. Su apellido se propuso, resultando el más votado y teniendo además la suerte de ser sorteado. Su teniente fue el doctor Morejón y Gato, quien recibió más votos que las familias Cárdenas y Herrera juntas; fue profesor de Universidad y es ahora conocido por haber publicado una obra sobre el azúcar. Se puede suponer que una probable concertación entre los hacendados permitió la entrada a este organismo de personas que, por su prestigio, su poder y su influencia, podían contribuir al éxito de todos.

La composición de la Junta en 1806 contiene cuatro veces el nombre de Pedroso, y los lazos familiares entre los personajes son muy cercanos. Esto no significa que esta familia hubiera sido la más poderosa de todas, sino que fue la más afortunada. En la propuesta para elegir un consejero, Ignacio Pedroso y Barreto obtuvo cuatro votos, quedando en tercera posición, ex aequo con el conde de O'Reilly; detrás se ubicaron Ignacio de Herrera con cinco votos, y Andrés Jáuregui, con nueve. Los nombres de los cuatro elegidos fueron puestos en una urna, saliendo electo Pedroso y Barreto. En este mismo escrutinio, para una plaza de consejero, Andrés Jáuregui superó por un voto a Francisco Javier de Pedroso y, en el segundo sorteo, nuevamente la suerte se inclinó a favor de los Pedroso.

Evocando a los Jáuregui hay que subrayar una cosa: el apellido, clasificado antes entre los comerciantes, lo sería entonces entre los hacendados. Andrés de Jáuregui y Arrostegui era hijo de Juan Tomás Jáuregui y Mayora, el primer cónsul ya mencionado. Se puede suponer que los Jáuregui realizaron el sueño de todo gran comerciante del Antiguo Régimen: hacer fortuna en el negocio para invertir en fincas. Aunque no generaba tanto dinero como los negocios, el prestigio conferido por la posesión de muchas hectáreas no tenía precio. La consagración del cambio de "clase" se expresó cuando Andrés Jáuregui a los cincuenta y seis años se casó con una Arrostegui y Herrera. Esto es un caso único en el periodo estudiado. En efecto, se puede hablar de dos líneas, la de los hacendados y la de los comerciantes, que no se mezclan. 
Merece una especial mención el caso de la familia Arango. Su principal representante, Francisco, mantuvo el puesto clave por un periodo amplio y cuya permanencia dependía únicamente de la Monarquía, con la cual se presume estaba en los mejores términos. Su hermano Ramón era el contador: manejaba los fondos y era inamovible. Cuando muere en 1798, otro hermano, Ciríaco, fue quien le sucedió, siendo electo por unanimidad. Ningún otro Arango quiso una plaza de prior o de consejero, parece que la estrategia de la familia Arango consistió en no mezclarse en eventuales rivalidades.

Por lo tanto, la apertura del Consulado a hacendados no relacionados directamente con familias tradicionales existió, pero fue muy rara. En 1806 después de once años de ejercicio, solamente cuatro de estos personajes son consejeros, pero ninguno prior. Hay una repartición más o menos concertada de las plazas en el seno de cada clase, atenuada por la parte de suerte introducida en cada elección. Una de las ventajas de este sistema, en el cual el número de plazas es definido y equitativo entre hacendados y comerciantes, es que incita a una buena armonía en el grupo constituido.

\section{Un buen entendimiento colectivo}

La separación de líneas matrimoniales entre hacendados y comerciantes no implica una separación económica. La distinción entre dos clases -es el término usado en la época- entre hacendados y comerciantes, tal como lo hizo la Monarquía, es bastante artificial. Francisco de Arango y Parreño dijo, el 4 de junio de 1804, que "estaban las dos clases tan unidas en los intereses generales de la Ysla que apenas se contaba comerciante distinguido en el pueblo que no fuese al mismo tiempo poseedor de uno o más Yngenios". En efecto, los intereses de hacendados y comerciantes eran los mismos: si los hacendados producían mucho azúcar, tabaco o café, los comerciantes ganaban exportándolos e importando todo lo necesario para su cultivo. Esta comunidad de intereses hacía reinar la mejor armonía a pesar de la separación en clanes de los dos grupos: el buen entendimiento favoreció los negocios. La lectura de las actas de las sesiones muestra pocos puntos de desacuerdo y aún menos disputas entre los dos grupos. La casi totalidad de las resoluciones fueron adoptadas por unanimidad. 
Las actas de las sesiones del Consulado son poco explícitas sobre las actividades de los comerciantes y menos aún sobre las de los hacendados; pero confirman la buena colaboración que caracterizaba las relaciones entre ellos. Por ejemplo, en abril de 1798, Nicolás Calvo de la Puerta y O'Farrill, estaba tan orgulloso de su ingenio Nueva Holanda, de concepción ultramoderna, que invitó oficialmente a todos los miembros del Consulado a visitarlo. También se hablaba de manera abierta de una nueva especie de caña que parecía dar más rendimientos, la de Otahiti. El Consulado era tan abierto que comunicaba por la prensa las noticias que podían favorecer la agricultura. Así, cuando en 1802 son recibidas dos arrobas de añil de Guatemala, se publicó un anuncio ofreciéndolas a quien se interesara en cultivarlo. No se puede hablar de competencia económica dentro de la oligarquía, sino de colaboración.

Para lograr esta colaboración, el trabajo era lógicamente repartido entre los consejeros según las competencias reconocidas o supuestas, primero, de cada una de las clases, y después de los individuos. Por lo tanto, toca a los hacendados el estudio de las mejoras agrícolas y a los comerciantes la resolución de los problemas económicos. Durante cierto tiempo José Ricardo O'Farril era el experto técnico del Consulado y estuvo encargado de redactar los informes sobre todas las nuevas máquinas presentadas como susceptibles de mejorar la producción. Y fueron los comerciantes Manuel González de Villarroel y Francisco Hernández, los que estudiaron la proposición hecha el 6 de noviembre de 1805 por José María de Quintana de fundar un banco en La Habana.

Se puede suponer que esta repartición del trabajo según las competencias, además de la eficacia que procuraba en la realización de las tareas, contribuyó al buen entendimiento colectivo. Por otra parte, ningún consejero parece haberse impuesto como personaje dominante por sus competencias, sus relaciones o su caudal. La autoridad y los honores quedan en manos del prior y de los cónsules. El único signo honorífico conferido a un particular lo fue a título póstumo. Cuando murió en agosto de 1795 el primer prior, el conde de Casa Montalvo, quien había participado en la fundación de la Junta con Arango, se decidió colocar su retrato en la sala de la Junta. Conviene subrayar el trabajo incansable y muy eficaz del síndico. Cumplió escrupulosamente su papel impulsando los debates y proponiendo muy hábilmente las 
mejores soluciones. Su trabajo fue apreciado por todos y parece que se respetaba más aún a la persona que a la función que ejercía.

La cohesión, que se apoya en intereses económicos, familiares y tal vez de amistad, confirió a la Junta una gran fuerza y revela también la importancia que tuvo la institución: si no hubiera sido tan importante, no hubiera quedado en manos de algunas personas. Y fue gracias a esta cohesión que los oligarcas pudieron utilizar al máximo los poderes confiados a la institución. Esto permitió un juego muy activo en la isla.

\section{Un juego sutil y benéfico}

El Consulado fue una institución que adquirió su lugar entre otras instituciones ya presentes en la colonia o en la metrópoli. Conviene, entonces, reflexionar sobre las relaciones directas entre la Junta de Gobierno y la Monarquía.

\section{El Real Consulado y su rey}

El Consulado de La Habana existía porque así lo había querido el rey de España. Nunca, a pesar de la distancia o del poder financiero y político de la oligarquía, se discutió este orden jerárquico. Al contrario, se trataba de aprovecharlo. Si la Junta reconocía en el rey de España a su señor, también esperaba que éste la protegiera como a su vasallo. La Monarquía buscaba a cambio fidelidad absoluta y ayuda económica en caso de necesitarla. Esto suponía discusiones y negociaciones entre los oligarcas y la Corona, aunque éstas no aparecieron por primera vez con el Consulado. Ya habían sido importantes en 1763, cuando Campomanes quiso imponer profundas reformas en la isla, pero tomaron una nueva dimensión con los objetivos asignados a la Junta. El Consulado sabía que se jugaba en Madrid, en gran parte, el porvenir de la colonia. Entonces, desde el 5 de agosto de 1795, se tomó la decisión de nombrar un apoderado en la Corte. Si bien la representación fue ocasional al principio, ésta se volvió permanente a partir de 1798. El primer apoderado se llamaba Matías Duende, nombrado a propuesta de Francisco de Arango y Parreño, quien lo presentó como merecedor de toda su confianza. A su muerte en 1799, le sucedió Francisco de Rucabado. La misión del apoderado consistía en defender los asuntos del Consulado directamente delante de los minis- 
tros e informar a la Junta de los proyectos en discusión o que hubieran podido tener una influencia en Cuba. Entonces, esta misión se asemejaba a la ordinariamente asignada a un embajador.

Lamentablemente las fuentes son poco explícitas sobre las sumas dadas a este apoderado, que, de tenerlas, nos darían indicaciones sobre su influencia. Lo que es seguro es que el puesto era atractivo. Cuando murió Matías Duende varias personas se presentaron espontáneamente para sucederle. El 27 de enero de 1796, Francisco de Arango y Parreño dijo que tener un apoderado en Madrid no bastaba, y añadió que la Junta debía: "Tener una persona condecorada, acreditada con el ministerio y con este público, tanto por su carácter como por su talento y por los servicios mismos que hubiese hecho a esta isla; quien pudiese apoyar en la Corte las ideas de la Junta." Arango propuso como consejero de Estado a Francisco de Saavedra, lo que fue aceptado por sus colegas. Rucabado debía obedecer a Saavedra. Por un asunto importante como el tráfico negrero, Francisco de Arango y Parreño, aumentó aún más el batallón con los oficios de su amigo, el conde de Mopox, quien era asimismo amigo personal de Manuel Godoy, el Príncipe de la Paz. El poder de la red era signo también del poder de la oligarquía.

Lo que parece nuevo en las relaciones entre el rey y la oligarquía es el hecho de que esta última tomara la iniciativa de proponer las reformas que debían permitir al rey desempeñar su papel natural de protector, árbitro y mediador. El papel de protector debía manifestarse por la dispensa de gracias a favor de la agricultura y el comercio. Siendo así, o Carlos IV fue un buen protector o bien Francisco de Arango y Parreño era muy atendido en la Corte. Como establecía una real orden del 26 de febrero de 1796, se permitió a los hacendados refinar ellos mismos el azúcar, obteniendo ventaja del endulzante blanco que tenía mucho más valor que el quebrado. Otra real orden, del 15 de octubre de 1800, renovó por diez años las excepciones de impuestos acordadas para ciertas exportaciones de café, algodón o de añil. Las gracias más determinantes fueron las concernientes a la importación de esclavos. Anteriormente, por un corto tiempo, entre 1789 y en 1791, la Monarquía había tomado medidas liberales. Pero en 1797 el Consulado se quejó de que el crecimiento de las plantaciones es tan importante que faltan brazos para cultivarlas: el sistema pareció fragilizado. El 26 de enero de 1804 el apoderado en la Corte estaba 
orgulloso de escribir al Consulado que la libre introducción de negros estaba permitida por doce años a nacionales, y por seis a extranjeros. Hacía tiempo que la Monarquía estaba a favor de la trata en La Habana (Tornero Tinajero 1996: 24), pero tal vez no quiso conceder privilegios que hubieran dañado a los comerciantes peninsulares, hasta no encontrar en Cuba una necesidad tan grande que los justificara. En el fondo, este privilegio no era nada más que la legitimación de un existente contrabando a gran escala.

Más generalmente, el rey era el árbitro supremo en toda materia, incluso comercial: era quien entregaba o negaba los privilegios, que constituía uno de los pilares del Antiguo Régimen. A veces ocurría que para tener rápidos ingresos, o para agradecerle a un súbdito que había hecho grandes servicios, el rey vendía o entregaba una licencia exclusiva de comercio con La Habana, dañando con ello a quienes no se beneficiaban con la licencia, y también a los consumidores, que tenían que hacerle frente a un único proveedor que podía imponer su precio. La Monarquía concedió varias veces privilegios para comerciar en Cuba, a pesar de las quejas del Consulado que "siempre mira todo lo que se llama estanco o privilegio con sentimiento de aversión y dolor". ${ }^{8}$ Se entienden aún mejor estos lamentos cuando se sabe que la economía de la isla estaba cada vez más especializada y por lo tanto cada vez más dependiente de los mercados exteriores, lo mismo por sus importaciones como por sus exportaciones. Este derecho de conceder privilegios era una temible arma de control por parte del rey. Se puede suponer que se sirvió hábilmente de ella cuando concedió al conde de Mopox, el 20 de julio de 1802, el permiso para la introducción de harinas que necesita prioritariamente La Habana. En efecto, Joaquín María Beltrán de Santa Cruz y Cárdenas, conde de Mopox, hermano político del segundo conde de Casa Barreto, cuñado del marqués de Casa Peñalver, casado con una Montalvo y O'Farrill, era al final de cuentas uno de los miembros más representativos de la oligarquía isleña. Concediendo privilegios a un miembro en particular, la Monarquía podía fragilizar las alianzas naturales de la oligarquía. No era al rey a quien había que enseñarle el proverbio "divide y reinarás". El Consulado no paraba de hacer informes muy documentados para mostrarle al rey todos los perjuicios que ocasionaba esta política a la 
isla y también al imperio. Pero no sería escuchado antes de 1818; entre tanto, tendría lugar un intenso contrabando (Moreno Fraginals 1995: 154).

Estas quejas, por supuesto, eran hechas con el debido respeto, quizás sincero, y más aún con un buen cálculo político, dado que la Junta necesitaba la protección del rey. En efecto, la influencia de éste superaba las fronteras de su imperio. Así, en un primer momento, la Junta pensó que la mejor manera de tener esclavos sería comprarlos a los portugueses. Sin embargo, una ley lusitana de 1751 impedía a los negreros de esta nación vender su "mercancía" a los extranjeros. En ese mundo fuertemente jerarquizado, el prior de la Junta de Fomento de La Habana por supuesto no tenía el rango suficiente para negociar con el rey de Portugal, así como tampoco lo tenía el capitán general, pero sí el rey de España. Entonces Arango, el 2 de diciembre de 1795, propuso escribir al rey para que pidiera a su homólogo portugués levantar esta prohibición. La misma diligencia, también a propósito de esclavos, fue adoptada al año siguiente con el papa. En efecto, los hacendados querían la dispensa del ayuno para los negros en los días de su observancia religiosa, para que ésta no afectara el rendimiento del trabajo, decisión que no podía ser tomada sino por el sumo pontífice.

La importancia que dio el Consulado a la necesidad de mantener lazos con la Corte muestra que uno de los objetivos iniciales, el de atar más estrechamente la isla con la península, se estaba realizando. El desarrollo de la trata negrera, el arbitrio sobre los privilegios y el trabajo de los esclavos, muestran hasta que punto la administración real desempeñaba un papel importante en la buena marcha de los negocios. Entre La Habana y Madrid el entendimiento durante el periodo estudiado era muy bueno. Esto se marca en la piedra y en el papel: la Junta decidió en 1803 erigir en La Habana una estatua de Carlos III en reconocimiento a los favores que recibió la colonia de la Monarquía; esta última envió cartas al Consulado manifestándole su satisfacción. Tener buena fama y buena influencia en Madrid era fundamental para la oligarquía. Pero también había que tener las mejores relaciones con los representantes del rey en la colonia. 


\section{Relaciones con las principales autoridades de la isla}

Los más altos representantes de la Monarquía en Cuba eran el capitán general, el intendente y el comandante de Marina. El capitán general era a la vez jefe político, primero de los militares y de los jueces, y vice-patrono de toda la isla: era, en breve, el teniente del rey. Eso tiene aún más importancia en un tiempo en el que la más rápida de las naves necesitaba entre dos y tres meses para traer desde la Corte las órdenes del monarca: en caso de crisis, había que poder contar con él para tomar decisiones de emergencia. De 1795 a 1807 , se sucedieron tres capitanes generales: Luis de las Casas (1793-1796), el conde de Santa Clara (1796-1799), y el marqués de Someruelos (1709-1812), todos muy entusiasmados de trabajar con la Junta.

La defensa del Real Consulado era una de las atribuciones del capitán general, y la Junta lo recordaba sin reserva si lo necesitaba. Se ve por ejemplo en junio de 1804, cuando el diputado de la Junta para Santiago fue encarcelado por el alcalde ordinario de dicha villa por un motivo desconocido. Los hacendados y los comerciantes pidieron explícitamente al capitán general que interviniera en este asunto: "Ya sea como protector de este cuerpo, ya sea como supremo magistrado." Como el propio rey, el capitán general podía también ser un mediador. En su calidad de vice-patrón de la isla pidió al obispo, en nombre de los hacendados, que se pudiera decir la Misa a los esclavos en los oratorios de los campos y no en las parroquias, transmitiendo la misma petición al rey. El objetivo aquí era evitar una concentración demasiado grande de esclavos que pudiera ser difícilmente controlable. Esta mediación fue eficaz ya que el obispo accedió a este deseo el 3 de agosto de 1799, y el rey confirmó la decisión en 1801.

El capitán general debía también vigilar y animar las tareas y a los miembros de la Junta. Para eso disponía de un medio particularmente eficaz, ya mencionado anteriormente: el de recomendar al rey las personas que tuvieron un celo particular en el puesto para el que fueron elegidas. El capitán general redactó el informe definitivo después de haber leído el informe del síndico. Un expediente del año 1796 resulta particularmente ilustrativo. ${ }^{9}$ Presenta simultáneamente el informe de Francisco de Arango y Parreño para Luis de las Casas y el de este último para Diego de Gardoqui. En este documento, Francisco de Arango

9 AGI, Estado 5, exp. 81. 
y Parreño clasifica a sus colegas según tres criterios: los que tuvieron una gran actividad e invirtieron su dinero para los éxitos del Consulado; los que fueron muy celosos, pero no dieron ningún real; y los que cumplieron su deber; en total, diez personas. El capitán general se contenta con señalar los méritos de los que terminaron sus periodos, en total cinco personas. Se muestra de acuerdo con el síndico, y a pesar de que no hace distinciones sobre los méritos, propone a la Corona recompensas adecuadas. El segundo cónsul, Lorenzo de Quintana, merecía, según él, la pequeña cruz de la orden de Carlos III, igualmente que Antonio de Arregui. Nicolás Calvo de la Puerta y O'Farrill, hacendado riquísimo, que invirtió grandes sumas en investigaciones agrícolas, merecía la plaza de prior honorario perpetuo, vacante desde la muerte del conde de Casa Calvo. Las Casas recomendó también a Pablo Boloix para un puesto en la Secretaría de Hacienda, al que aspiraba, también por su servicio sin ningún salario en la Secretaría de Luisiana y Florida durante dos años y medio. Tal y como Francisco de Arango y Parreño sugería, Las Casas escribió que el marqués de Arcos no debía ser incluido en la primera promoción de la gente agraciada. En cambio, recomendaba calurosamente a la Monarquía al autor del informe adjunto, es decir al propio síndico, Francisco de Arango y Parreño. Decir que Luis de las Casas hace un elogio de Francisco de Arango y Parreño sería poco, digamos que lo presenta como un estadista. El futuro le daría la razón.

La entrada al Consulado no representaba solamente la marca de un reconocimiento para sus miembros, sino también un trampolín social. Ciertamente, la capacidad de maniobra de la que disponía el capitán general, en conjunto con la autoridad de la que estaba investido contribuyeron a lograr las buenas relaciones entre éste y la Junta. Esta gracia se aprecia al considerar tan sólo las medidas tomadas a favor de la oligarquía. Una de las primeras demostraciones de esta toma de responsabilidad de parte de un capitán general se verificó en 1798 , cuando expiró una real autorización que permitía a los neutrales el tráfico de esclavos. El Consulado solicitó al conde de Santa Clara la prórroga de esta autorización hasta que llegara una orden real confirmándola o prohibiéndola. El marqués de Someruelos fue más lejos aún $\mathrm{y}$, al asumir sus funciones, no puso en práctica la orden real del 10 de abril de 1799 que suspendió el abastecimiento de la isla por los navíos neutrales (que eran en su mayoría estadounidenses). Expuso 
que la supervivencia de la isla en ese tiempo de guerra, en el que la marina española no podía atender sus deberes, dependía del incumplimiento de la real orden. Los comerciantes españoles se escandalizaron, pero la Monarquía concedió razón a su representante y a partir de 1800 la flota de Estados Unidos alcanzó, por mucho tiempo más, el primer rango en el tráfico con Cuba. La Monarquía aceptó sabiamente los hechos consumados. Sin embargo, no fue el caso de los comerciantes gaditanos quienes, frustrados por dejar la plaza a los recién llegados, respondieron con una cierta oposición entre ambos consulados. Habría que investigar si este asunto es un indicador de la rápida criollización de los mercaderes instalados en La Habana, quienes pidieron comerciar con Estados Unidos en contra de los intereses de las casas de comercio peninsulares.

Frente al capitán general, la treintena del Consulado era consciente de su propia fuerza. A veces el representante del rey llamaba a la generosidad de la gente cuya reunión semanal presidía. En la sesión del 5 de marzo de 1806, se leyó una real orden pidiendo al capitán general que incitara a los vecinos y las casas de comercio a efectuar los más grandes préstamos a la Monarquía para financiar la guerra contra Gran Bretaña. Luego de pintar una triste situación de España después de Trafalgar, Someruelos rememoró las beneficencias acordadas, particularmente en el ámbito comercial, concluyendo que el Consulado estaba en la obligación de llevar a cabo esfuerzos extraordinarios, aunque ya desde la declaración de guerra había anticipado los deseos de su Majestad. Obedeciendo esta demanda, la Junta nombró una comisión para reunir el dinero, misma que fue rápidamente disuelta. En efecto, sobrevino un real decreto del 26 de julio que aumentaba para todo el imperio los derechos de importación. La suma a recaudar debía lograr reembolsar un préstamo de cien millones de reales contratado por la Monarquía con el Consulado de Cádiz. Entonces, como por una parte el rey decidió levantar impuestos sin negociaciones, y por la otra este dinero debía terminar en las cajas del Consulado de Cádiz, se optó por no cumplir lo que pedía el capitán general. Este asunto muestra tanto la buena voluntad de la Junta, que al principio era partidaria de colaborar, como su poder de decir no al capitán general. Sin embargo, cuando Francia invadió España en 1808, la oligarquía contribuyó económicamente. Se puede ver en los informes para peticiones de títulos de Castilla, cuánto dio cada uno de sus miembros, a veces en azúcar. 
También esto es muy significativo: se daba dinero, pero de una forma u otra se presentaba la cuenta más tarde.

Además el capitán general, con todo lo poderoso que hubiera podido ser, era también un funcionario deseoso de hacer una buena carrera y las recomendaciones de la poderosa oligarquía habanera podían quizás serle de utilidad. Entonces, el Consulado no se privaba de escribir al rey para informarle sobre sus sentimientos con respecto al primer jefe de la isla: siempre eran muy positivos. La Junta tenía por regla manifestar su opinión sobre los capitanes generales al dejar éstos su cargo, para que el informe no estuviera marcado por el temor o la adulación. Sin embargo, cuando Someruelos presidió por primera vez la Junta, el 22 de mayo de 1799 , se le recordó esta regla, lo cual era una manera de mostrar al recién llegado su influencia. Cuando en 1796 Luis de las Casas debió dejar el mando por razones de salud, se fue con las felicitaciones oficiales y ceremoniosas del Consulado, mediante una diputación representada por el marqués de Cárdenas de Montehermoso y Pedro Juan de Erice.

La autoridad del capitán general tampoco era cuestionada, la asamblea de los hacendados y de los comerciantes se cuidaba siempre de tener buenas relaciones con él. Esta gran armonía que reinaba entre el Consulado y su presidente era muy natural: la primera misión del capitán general era mantener una buena administración de la isla y la Junta económica y de Gobierno había sido creada por eso. Como se ha dicho, su poder podía ser útil a la oligarquía. Se puede suponer que los lazos entre los hacendados y comerciantes con el jefe de la isla no se limitaban a las reuniones del Consulado. Invitaciones, bailes, que se traducían quizás en lazos de amistad, debían acercar aún más a los criollos a este funcionario. Quizás el mejor ejemplo es el dado por Luis de las Casas, quien invirtió en un ingenio y cuya hermana se casó con el primer conde de O'Reilly. Ninguno de los dos era criollo, pero de esta unión procedió la grande y rica línea de los O'Reilly de Cuba.

Las relaciones entre la Junta de Fomento, por un lado, y el intendente y el comandante de Marina, por el otro, no eran tan sencillas como las habidas con el capitán general. Las cuestiones financieras estaban aquí más presentes, y se inscribían dentro de confrontaciones de intereses, por lo cual las negociaciones eran más numerosas y complejas. Sin querer hacer un estudio de la Intendencia habanera (Parcero Torre 1998: 259), recordamos que ésta fue la primera creada en 
América Latina en 1765; formando parte del conjunto de reformas desarrollado por la Monarquía después de la invasión inglesa. Su campo de acción principal era la Hacienda. También estaba asignado a esta institución borbónica por excelencia el mantenimiento de la defensa (reales ejércitos y murallas) y la compra de la producción de tabaco, dado que hasta 1817 este último era un monopolio real. El comandante de Marina tenía a su cargo todo lo referido a la actividad naval, desde la construcción de navíos hasta la protección costera. Esto era importantísimo en Cuba porque los intercambios se hacían a través de barcos, y había una feroz guerra de corso en esta época en el Caribe.

En junio de 1795, por ejemplo, el intendente negó al Consulado la aplicación del derecho de avería que se le había concedido por las mercancías hasta entonces exentas de todos los impuestos. También el comandante de Marina se opuso a los hacendados sobre lo relativo a la tala de árboles, que buscaba reservar para la construcción de navíos. El aumento de la producción de caña implicaba quemar árboles para obtener después terrenos fértiles, y leña para calentar las calderas (Moreno Fraginals 1978: 74). Sobre estos dos asuntos, tanto como sobre otros, el Consulado salió vencedor. Lo anterior, por una sencilla razón: la oligarquía jugaba siempre la misma carta, la del dinero. No es que comprara a los hombres, pero sin dinero no se podía hacer la guerra, y tanto el intendente del Ejército como el comandante de Marina lo sabían, por supuesto, perfectamente.

Los fondos que necesitaban, el situado, venía de las cajas de México; pero en tiempo de guerra llegaba más irregularmente. Durante el periodo intermedio entre la llegada de un situado y el siguiente, el intendente y comandante debían pedir al hacendado, o al comerciante, o al organismo que los representaba, la concesión de algunos préstamos. Las sumas pedidas a veces eran faraónicas, lo que muestra el grado de potencia que se suponía, con o sin razón, que tenía la élite. Por ejemplo, el 22 de diciembre de 1802, Antonio María Arturo, el comandante de Marina, pidió un préstamo de 240.000 pesos. El Consulado acordó por unanimidad 25.000 pesos, ni uno más. No se sabe cuál era el interés, ni siquiera si lo había. El $1^{\circ}$ de agosto de 1804 , el intendente Luis de Viguri pidió 190.000 pesos para comprar la cosecha de tabaco. Se dirigió al Consulado, después de haber recaudado solamente 22.000 pesos de los particulares. Después de algún tiempo, 
una comisión logró reunir la suma solicitada, una asociación de comerciantes dio más del $50 \%$ del total, y el Consulado, ayudado por sus sucursales en toda la isla, participó con un $25 \%$.

De todos los asuntos comunes de la Junta de Fomento y las autoridades militares, hay uno que no se puede callar, por las fuertes consecuencias que trajo aparejadas. Nos referimos a la participación del Consulado en la defensa de la isla. Ya a fines de 1799, la Junta participó en el mantenimiento de los torreones costeros para impedir desembarcos de piratas. Pero, sobre todo, a fines de 1805 decidió entregar 36.000 pesos al comandante de Marina para la duplicación del número de lanchas cañoneras destinadas a la protección de la isla y de los buques cargados de azúcar.

En cambio, los representantes de la Corona podían complacer a la Junta. El intendente apoyó las gestiones con la Monarquía, que acordó en 1803 que los comerciantes tuvieran derecho a exportar el azúcar hacia los Estados Unidos bajo banderas neutrales. El Consulado agradeció este gesto, pero sugirió que el derecho concedido a la explotación de azúcar fuera completado por un permiso de importación de otros productos provenientes de Estados Unidos. Asimismo, cuando la Junta estaba satisfecha con el trabajo del intendente, también hacía su elogio al rey. Un punto de convergencia de los intereses era siempre buscado, a veces más activamente. Así, en junio de 1805 el Consulado, asombrado por el silencio de la Real Hacienda relativo a las reformas que proponía, pensó que éste se debía a una sobrecarga de trabajo. La Junta propuso entonces, quizás con un poco de ironía, tomar a su cargo el sueldo de algunos empleados suplementarios para que el trabajo fuera más de prisa.

La Junta de Fomento del Real Consulado mantenía entonces una relación constante y estrecha con las primeras autoridades de la isla. Necesitaba su apoyo y su benevolencia para lograr sus fines. Si podía pedir y obtener ayuda, era porque sabía que también podía prestar grandes servicios a cambio. Habría que añadir que la fuerza del Consulado provenía igualmente de su posición frente a otros centros de poderes insulares criollos. 
Relaciones con los centros de poder insulares criollos

No es necesario detenerse mucho tiempo en las relaciones entre el Consulado y el Cabildo, por dos razones: la primera, porque las tareas asignadas a las dos instituciones eran en gran parte diferentes, y la segunda, porque la mayoría de los hacendados se encontraban en el Cabildo, por lo que había poca colaboración y ningún conflicto. Como comparación con el cuadro 2 , presentamos una parte de la composición del Cabildo de 1803 (cuadro 3).

Cuadro 3: Las funciones principales del Cabildo de La Habana, $1^{\circ}$ de enero de 1803

\begin{tabular}{|c|c|}
\hline Cargo & Titular \\
\hline Alcaldes ordinarios de La Habana & $\begin{array}{l}\text { Conde de O'Reilly } \\
\text { José de Garro y Zayas }\end{array}$ \\
\hline Regidor alférez real & Francisco de Arango y Parreño \\
\hline $\begin{array}{l}\text { Regidor alcalde mayor provincial } \\
\text { de la Santa Hermandad }\end{array}$ & Adriano de la Cruz \\
\hline Regidor fiel ejecutor & Ciríaco de Arango \\
\hline Regidores o tenientes de regidores & $\begin{array}{l}\text { Sebastián Peñalver Barreto } \\
\text { Francisco de Peñalver } \\
\text { Gonzalo Herrera } \\
\text { Joaquín de Herrera } \\
\text { Luis Ignacio Cavallero } \\
\text { Teniente coronel Antonio de la Luz } \\
\text { Carlos Pedroso } \\
\text { Francisco Loinaz y Lizundia }\end{array}$ \\
\hline
\end{tabular}

Fuente: Actas capitulares del ayuntamiento de La Habana, trasuntadas, libro 59, $\mathrm{AOHCH}$.

Las funciones más importantes del Cabildo, reunidas en el cuadro 3, eran hereditarias, a excepción del prestigioso puesto de alcalde ordinario, que era anual. Se puede ver cómo algunas familias -Peñalver, Herrera, Pedroso, O'Reilly, Arango- monopolizaban los cargos. Habría que añadir que Andrés de Jáuregui, teniente de síndico del Consulado, era también en el año 1803 síndico del Cabildo. Otra vez la familia Arango estaba muy bien representada: Francisco desempeñaba a partir de ese año el puesto clave de regidor y alférez real, y Ciríaco fue electo nuevamente por unanimidad, regidor fiel ejecutor. 
Desempeñando así Francisco de Arango y Parreño los dos cargos fundamentales del Consulado y del Cabildo, se puede suponer que los asuntos de la oligarquía fueron despachados más rápidamente que los de la Intendencia. Sin embargo, la colaboración se inscribía a veces en un cuadro oficial, particularmente las cuestiones de obras y de empedrado.

La Junta de Fomento y el Cabildo tendrían una magnífica ocasión para trabajar juntos después del incendio del 25 de abril de 1802, que destruyó los barrios extramuros de La Habana. Este incendio dejó a mucha gente en la calle, haciendo imposible alojarla nuevamente en la ciudad, por falta de materiales. Francisco de Arango y Parreño, con el cinismo que le caracterizaba, señaló que si no alcanzaba en La Habana la madera para fabricar cajas de azúcar, por supuesto que no había suficiente para edificar casas. A lo que añadió que lo mejor sería fundar una nueva villa para fijar a aquélla población de pobres, lo que permitiría descongestionar La Habana y favorecer la implantación de blancos en el campo: ya que, desde hacía algunos años, la introducción masiva de esclavos negros empezaba a suscitar inquietudes. Además, decía, se podría tratar de comprar la madera necesaria a neutrales, y vender a cambio azúcar. El documento no aclara si parte de estas maderas compradas podrían servir para hacer cajas de azúcar. Arango asumió personalmente el asunto, y apoyándose en el Cabildo y la Junta de Fomento, hizo emerger una nueva ciudad: San Marcos.

La principal ayuda que recibió el Consulado en el fomento de la isla vino de una institución muy emblemática del Siglo de las Luces, la Sociedad Económica de Amigos del País. La creación de la Sociedad Económica de Amigos del País de La Habana precedió a la del Consulado sólo por algunos meses, celebrando su primera sesión en enero de 1793. También constituyó una especie de prefiguración de éste, dado que la asociación tenía por objetivo el progreso y la difusión de las ciencias en la isla (González-Ripoll Navarro 1999), agrupando en su origen a un pequeño núcleo de gente, que formaba parte de la mejor sociedad. ${ }^{10}$ La entrada a la Sociedad Económica de Amigos del País no estaba reservada a hacendados o grandes comerciantes, pero por supuesto fueron ellos los primeros en entrar. La creación 10 Actas de la Sociedad Económica de Amigos del País, libro 1, Instituto de Litera-
tura y Linguística de La Habana. 
del Consulado poco después, en gran medida tomaría a su cargo el mismo objetivo, amplificándolo. La colaboración entre las dos instituciones fue, por lo tanto, natural y era sobre todo de orden financiero. Los dos organismos se asociaron para premiar la investigación científica a través de muchos concursos sobre temas muy variados. Por ejemplo, en enero de 1799, la Sociedad Económica de Amigos del País promovió un concurso dotado de dos mil pesos para la mejor memoria escrita sobre explotación de ingenios, y el Consulado participó con 500 pesos. Las dos instituciones se juntaron también para recompensar memorias sobre límites de propiedades y sobre enfermedades del campo. La Junta de Fomento se hizo de una reputación de mecenazgo en el ámbito de las ciencias que superaba los límites naturales de la isla. Así el 29 de mayo de 1804 un inglés de Jamaica, Brian Higgins, hizo referencia a sus invenciones y observaciones concernientes al café, a los combustibles y al ron. Se incluyeron también proyectos de mayor envergadura, como la creación de un jardín botánico y la formación de un joven criollo, José Esteves, en Madrid, en las ciencias naturales.

El Consulado favoreció también proyectos sobre infraestructuras de carreteras y comercio. Por ejemplo, el 14 de julio de 1802 se discutió el aplanamiento de la colina de Nazareno, punto difícil de pasaje obligado para las gruesas carretas cargadas de cajas de azúcar. El 7 de diciembre de 1802 se empezó la confección de la primera carta topográfica de la isla, con el concurso esta vez de los representantes del rey. Se habló también del fomento de una compañía de seguros marítimos con el objetivo de favorecer el comercio y particularmente la trata.

En este dominio de las ciencias hay que subrayar una vez más la acción preponderante de Francisco de Arango y Parreño. Su ingenio, La Ninfa, era un condensado de investigaciones e innovaciones tecnológicas. Sin embargo no era tanto producto de su propia inventiva, lo que presentaba regularmente ante los miembros del Consulado, sino la experiencia que había adquirido en el gran viaje que hizo con el conde de Casa Montalvo. En 1793 y 1794, estos dos amigos, recorrieron España, Portugal, Inglaterra, Barbada y Jamaica, buscando informaciones sobre progresos técnicos, agrícolas y el estado del comercio. El conde de Casa Montalvo murió pocos meses después de la primera sesión del Consulado, y Arango se quedó solo para presentar en cada 
sesión algo de los conocimientos adquiridos en este viaje. En noviembre de 1795 presentó un teodolito e instrumentos matemáticos comprados en Inglaterra; en diciembre, presentó el estado del comercio de Lisboa y de Cádiz. Desde octubre, Francisco de Arango y Parreño anunció que una máquina de vapor, encargada en Inglaterra, estaba a punto de ser enviada a La Habana. Este interés por la máquina de vapor, que se pensaba acoplar a los trapiches, es absolutamente destacable: muestra toda la modernidad y todo el deseo de progreso de la oligarquía habanera.

Es necesario añadir que el Consulado no defendía únicamente los intereses de la oligarquía habanera frente al capitán general y al intendente, sino también los de sus diputados repartidos en toda la isla. Las ciudades cubanas representadas eran primariamente puertos, y querían hacer negocios con pabellones neutrales y recibir navíos negreros. Comerciar y tener brazos para la agricultura: se entiende que estos deseos eran defendidos por el Consulado, y lo eran con éxito. De una manera más general, el Consulado a su vez se hacía el protector de todos los que podían ser útiles al fomento económico de la isla. Intervino por ejemplo a favor del negrero inglés Felipe Allwood en contra de la amenaza de expulsión que pesaba sobre él, y apoyó a los franceses que querían establecerse en la isla para sembrar café.

Resulta ahora fácil juzgar la eficacia de estas negociaciones con las autoridades y este estímulo a las ciencias. Basta considerar algunas cifras dadas por Alejandro de Humboldt (1998: 217 y 285). En 1795 por el puerto de La Habana, fueron exportadas 70.437 cajas de azúcar en contra de las 181.272 de 1807 ; esto sin contar con el desarrollo de la caña en otras regiones cubanas. El producto de la aduana habanera sumaba 640.583 duros en 1795 y 1.178 .974 en 1808 .

\section{Conclusión}

Este estudio fue construido esencialmente a partir de la lectura de las actas de sesiones del Consulado. La riqueza de estas fuentes hacen imposible desplegar en algunas líneas todos los aspectos de la institución. Además, pese a su precisión, hay partes que permanecen oscuras, como lo relativo a la suma y gestión de los fondos dados al apoderado en Madrid; o el detalle de las negociaciones en el seno de la oligarquía. Es necesario señalar que muchos aspectos de esta historia no 
aparecen por falta de fuentes, y es peligroso tratar de reconstruirlos por deducciones. Pero afortunadamente tenemos algunas certezas. Desde el punto de vista puramente metodológico, una confirmación, si es que era necesaria, es que no se puede entender una institución sin tomar en cuenta a las personas que la conforman. La actividad incansable y la personalidad de Francisco de Arango y Parreño, a la vez síndico del Consulado y regidor alférez real del Cabildo, son fundamentales para entender el desarrollo de la institución. En este sentido un estudio más amplio de la oligarquía que ponga de relieve los cargos en las milicias, o en otros ramos de la administración de la isla, permitiría a su vez un mejor entendimiento de su poder. Desde el punto de vista institucional aparece la Junta de Fomento como una especie de híbrido del Cabildo y de la Sociedad de Amigos del País. Del Cabildo tomaba el carácter cerrado que conserva el poder en las manos de algunas familias, y de la que ser parte podía permitir un ascenso social. De la Sociedad de Amigos del País reproducía la aplicación de principios cartesianos para favorecer un desarrollo económico. La originalidad del Consulado reside en la agrupación de hacendados y de comerciantes para defender intereses comunes. Es esto lo que confiere a la Junta su verdadera fuerza. Ahora, en efecto, los más altos intereses de la plantación y del comercio, se fusionan en una institución dotada de una cierta autonomía que tiene derecho de actuar en toda la isla. Por lo cual, el poder de cada miembro es a la vez multiplicado y legitimado dado que se ejerce dentro de una institución situada bajo el Patronato Real. Esta fuerza y esta legitimación van a llevar a la oligarquía, como estaba previsto, a tomar iniciativas y no solamente a reaccionar, como antes, frente a las decisiones reales.

Al final de los doce años de ejercicio, el balance financiero es positivo tanto para los oligarcas como para la Monarquía. Para los oligarcas, ya que muchos se convierten verdaderamente en sacarócratas, dado que las facilidades que obtienen para importar esclavos y exportar azúcar multiplican a una velocidad asombrosa la producción y por lo tanto su poder económico. Para la Monarquía también, porque recupera mediante impuestos una parte (demasiado grande según el Consulado) del dinero así generado. Hacía tiempo que la Corona estaba a favor de una cierta liberalización comercial en La Habana, pero quizás no dio los impulsos decisivos hasta no encontrar en Cuba una fuerza capaz de hacer competencia seria a Cádiz: el rey es el árbitro de 
los privilegios, y la política es un sutil juego de equilibrio de poderes. Precisamente, en 1807 el balance político general es más difícil de medir: España es incapaz de absorber todo el azúcar producido y de proveer a la isla en todas sus necesidades. De alguna manera el Consulado contribuyó a romper el lazo económico clásico entre metrópoli y colonia, cambiando necesariamente los datos políticos. De momento la oligarquía proclama una fidelidad absoluta a la Monarquía, notable por el reconocimiento de las ventajas concedidas y por las que continuará concediendo mediante condecoraciones y títulos de Castilla, de los que la élite estaba hambrienta. Éste es un punto importante. Si bien los oligarcas tienen una percepción revolucionaria de la economía, y se ubican en la vanguardia en lo relativo al progreso científico, mantienen una mentalidad de Antiguo Régimen muy fuerte, sobre la cual se apoya el rey de España. Este último asegura también su control emplazando sus regimientos al servicio de la oligarquía, para aplastar las eventuales sublevaciones de esclavos: el fantasma de Santo Domingo enturbiaría durante mucho tiempo las noches de los hacendados, más que la mala conciencia de la que carecían.

En efecto, el balance humano de estos doce años es espantoso: los grandes perdedores de las negociaciones fueron los millares de africanos arrancados de su tierra para ser inmersos en el mundo inhumano de la plantación esclavista. En un ámbito muy diferente, el balance ecológico es también dramático: el boom azucarero quemó literalmente gran parte de la fauna y de la flora de Cuba. La amplitud de estas catástrofes es otro testimonio del poder de la oligarquía. Por primera vez, con la Junta de Fomento, los hombres empezaron a organizar a gran escala el territorio en su provecho. Por otra parte, aunque los hacendados y los comerciantes favorecían sus intereses construyendo nuevos caminos para llevar el azúcar de los ingenios a los puertos, las carreteras al final servían a todo el mundo. Al invertir dinero en la defensa de los ingenios costeros, defendían también la isla. Quizás es por eso, y por el poder que ostentaban, que actuaron como si sus intereses fueran los de toda la isla, haciendo de ésta una entidad política. En 1802, con la conclusión de la paz de Amiens, los miembros del Consulado, agradecieron calurosamente al capitán general y al intendente por haberlos preservado de la miseria. 